\title{
On Certain Properties of Random Apollonian Networks
}

\author{
Alan Frieze, Charalampos E. Tsourakakis \\ Department of Mathematical Sciences, Carnegie Mellon University, USA \\ aflperandom.math.cmu.edu, ctsourak@math.cmu.edu
}

\begin{abstract}
In this work we analyze fundamental properties of Random Apollonian Networks [34]35], a popular random graph model which generates planar graphs with power law properties. Specifically, we analyze (a) the degree distribution, (b) the $k$ largest degrees, (c) the $k$ largest eigenvalues and (d) the diameter, where $k$ is a constant.
\end{abstract}

\section{Introduction}

Due to the surge of interest in social networks, the Web graph, the Internet, biological networks and many other types of networks, a large amount of research has focused on modeling real-world networks in recent years. Existing well-known models include the preferential attachment model [7], Kronecker graphs [28], the Cooper-Frieze model [16], the Aiello-Chung-Lu model [1], protean graphs [31] and the FabrikantKoutsoupias-Papadimitriou model [21]. In this work we focus on Random Apollonian Networks (RANs), a popular random graph model for generating planar graphs with power law properties [35]. Before we state our main results we briefly describe the model.

Model: An example of a RAN is shown in Figure 1 At time $t=1$ the RAN is shown in Figure 1a At each step $t \geq 2$ a face $F$ is chosen uniformly at random among the faces of $G_{t}$. Let $i, j, k$ be the vertices of $F$. We add a new vertex inside $F$ and we connect it to $i, j, k$. Higher dimensional RANs also exist where instead of triangles we have $k$-simplexes $k \geq 3$, see [34]. It is easy to see that the number of vertices $n_{t}$, edges $m_{t}$ and faces $F_{t}$ at time $t \geq 1$ in a RAN $G_{t}$ satisfy:

$$
n_{t}=t+3, m_{t}=3 t+3, \quad F_{t}=2 t+1 \text {. }
$$

Note that a RAN is a maximal planar graph since for any planar graph $m_{t} \leq 3 n_{t}-6 \leq 3 t+3$.

Surprisingly, despite the popularity of the model various important properties have been analyzed experimentally and heuristically with lack of rigor. In this work, we prove the following theorems using existing techniques [322[29].

\section{Theorem 1 (Degree Sequence).}

Let $Z_{k}(t)$ denote the number of vertices of degree $k$ at time $t, k \geq 3$. For $t$ sufficiently large and for any $k \geq 3$ there exists a constant $b_{k}$ depending on $k$ such that

$$
\left|\mathbb{E}\left[Z_{k}(t)\right]-b_{k} t\right| \leq K, \text { where } K=3.6 .
$$

Furthermore, for any $\lambda>0$

$$
\operatorname{Pr}\left[\left|Z_{k}(t)-\mathbb{E}\left[Z_{k}(t)\right]\right| \geq \lambda\right] \leq e^{-\frac{\lambda^{2}}{72 t}}
$$

For previous weaker results on the degree sequence see [33 35]. An immediate corollary which proves strong concentration of $Z_{k}(t)$ around its expectation is obtained from Theorem 1 and a union bound by setting $\lambda=10 \sqrt{t \log t}$. Specifically:

Corollary 1. For all possible degrees $k$

$$
\operatorname{Pr}\left[\left|Z_{k}(t)-\mathbb{E}\left[Z_{k}(t)\right]\right| \geq 10 \sqrt{t \log t}\right]=o(1) .
$$

The next theorem provides insight into the asymptotic growth of the highest degrees of RANs and is crucial in proving Theorem 3 . 


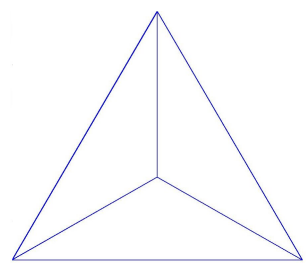

(a) $t=1$

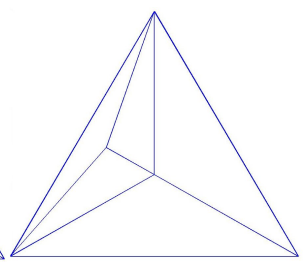

(b) $t=2$

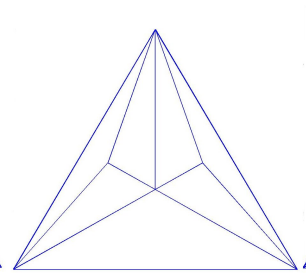

(c) $t=3$

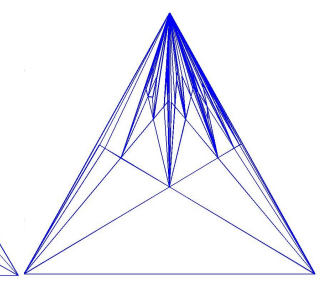

(d) $t=100$

Fig. 1: Snapshots of a Random Apollonian Network (RAN) at: (a) $t=1$ (b) $t=2$ (c) $t=3$ (d) $t=100$.

Theorem 2 (Highest Degrees). Let $\Delta_{1} \geq \Delta_{2} \geq \ldots \geq \Delta_{k}$ be the $k$ highest degrees of the RAN $G_{t}$ at time $t$ where $k$ is a fixed positive integer. Also, let $f(t)$ be a function such that $f(t) \rightarrow+\infty$ as $t \rightarrow+\infty$. Then whp

$$
\frac{t^{1 / 2}}{f(t)} \leq \Delta_{1} \leq t^{1 / 2} f(t)
$$

and for $i=2, \ldots, k$

$$
\frac{t^{1 / 2}}{f(t)} \leq \Delta_{i} \leq \Delta_{i-1}-\frac{t^{1 / 2}}{f(t)}
$$

The growing function $f(t)$ cannot be removed, see [22]. Using Theorem 2 and the technique of Mihail and Papadimitriou [29] we show how the top eigenvalues of the adjacency matrix representation of a RAN grow asymptotically as $t \rightarrow+\infty$ whp.

Theorem 3 (Largest Eigenvalues). Let $k$ be a fixed positive integer. Also, let $\lambda_{1} \geq \lambda_{2} \geq \ldots \geq \lambda_{k}$ be the largest $k$ eigenvalues of the adjacency matrix of $G_{t}$. Then whp $\lambda_{i}=(1 \pm o(1)) \sqrt{\Delta_{i}}$.

Also, we show the following refined upper bound for the asymptotic growth of the diameter.

Theorem 4 (Diameter). The diameter $d\left(G_{t}\right)$ of $G_{t}$ satisfies in probability $d\left(G_{t}\right) \leq \rho \log t$ where $\frac{1}{\rho}=\eta$ is the unique solution greater than 1 of the equation $\eta-1-\log \eta=\log 3$.

The outline of the paper is as follows: in Section 2 we present briefly related work and technical preliminaries needed for our analysis. We prove Theorems 1, 2, 3 and 4 in Sections 3, 4, 5 and 6respectively. Unavoidably due to the space constraint we have included for completeness reasons the proofs of certain lemmas which are omitted from the main part of our paper in the Appendix 8 In Section 7 we investigate another property of the model. Finally, in Section 8 we conclude by suggesting few open problems.

\section{Related Work}

Apollonius of Perga was a Greek geometer and astronomer noted for his writings on conic sections. He introduced the problem of space filling packing of spheres whose classical solution, the so-called Apollonian packing [25], exhibits a power law behavior. Specifically, the circle size distribution follows a power law with exponent around 1.3 [11]. Apollonian Networks (ANs) were introduced in [4] and independently in [20]. Zhou et al. [35] introduced Random Apollonian Networks (RANs). Their degree sequence was analyzed inaccurately in [35] (see comment in [33]) and subsequently using physicist's methodology in [33]. Eigenvalues of RANs have been studied only experimentally [5]. Concerning the diameter of RNAs it has been shown to grow logarithmically [35] using heuristic arguments (see for instance equation B6, Appendix $\mathrm{B}$ in [35]). RANs are planar 3-trees, a special case of random $k$-trees [27]. Cooper and Uehara [17] and Gao [23] analyzed the degree distribution of random $k$-trees, a closely related model to RANs. In RANs -in

\footnotetext{
$\overline{{ }^{1} \text { An event } A_{t}}$ holds with high probability $(w h p)$ if $\lim _{t \rightarrow+\infty} \operatorname{Pr}\left[A_{t}\right]=1$.
} 
contrast to random $k$-trees- the random $k$ clique chosen at each step has never previously been selected. For example, in the two dimensional RAN any chosen face is being subdivided into three new faces by connecting the incoming vertex to the vertices of the boundary. Random $k$-trees due to their power law properties have been proposed as a model for complex networks, see, e.g., [1724] and references therein. Recently, a variant of $k$-trees, namely ordered increasing $k$-trees has been proposed and analyzed in [30]. Closely related to RANs but not the same are random Apollonian network structures which have been analyzed by Darrasse, Soria et al. [8 18 19].

Bollobás, Riordan, Spencer and Tusnády [10] proved rigorously the power law distribution of the Barabási-Albert model [7]. Chung, Lu, Vu [15] Flaxman, Frieze, Fenner [22] and Mihail, Papadimitriou [29] have proved rigorous results for eigenvalue related properties of real-world graphs using various random graph models. In Section 3 we invoke the following useful lemma.

Lemma 1 (Lemma 3.1, [14]). Suppose that a sequence $\left\{a_{t}\right\}$ satisfies the recurrence

$$
\begin{gathered}
a_{t+1}=\left(1-\frac{b_{t}}{t+t_{1}}\right) a_{t}+c_{t} \\
\text { for } t \geq t_{0} \text {. Furthermore suppose } \lim _{t \rightarrow+\infty} b_{t}=b>0 \text { and } \lim _{t \rightarrow+\infty} c_{t}=c . \text { Then } \lim _{t \rightarrow+\infty} \frac{a_{t}}{t} \text { exists and } \\
\lim _{t \rightarrow+\infty} \frac{a_{t}}{t}=\frac{c}{1+b} .
\end{gathered}
$$

In Section 3 we also use the Azuma-Hoeffding inequality [6[26].

Lemma 2 (Azuma-Hoeffding inequality). Let $\lambda>0$. Also, let $\left(X_{t}\right)_{t=0}^{n}$ be a martingale with $\mid X_{t+1}-$ $X_{t} \mid \leq c$ for $t=0, \ldots, n-1$. Then:

$$
\operatorname{Pr}\left[\left|X_{n}-X_{0}\right| \geq \lambda\right] \leq \exp \left(-\frac{\lambda^{2}}{2 c^{2} n}\right)
$$

\section{Proof of Theorem 1}

We decompose our proof in a sequence of Lemmas. For brevity let $N_{k}(t)=\mathbb{E}\left[Z_{k}(t)\right], k \geq 3$. Also, let $d_{v}(t)$ be the degree of vertex $v$ at time $t$ and $\mathbf{1}\left(d_{v}(t)=k\right)$ be an indicator variable which equals 1 if $d_{v}(t)=k$, otherwise 0 . Then, for any $k \geq 3$ we can express the expected number $N_{k}(t)$ of vertices of degree $k$ as a sum of expectations of indicator variables:

$$
N_{k}(t)=\sum_{v} \mathbb{E}\left[\mathbf{1}\left(d_{v}(t)=k\right)\right] .
$$

We distinguish two cases in the following.

\section{- CASE 1: $k=3$ :}

Observe that a vertex of degree 3 is created only by an insertion of a new vertex. The expectation $N_{3}(t)$ satisfies the following recurrence $e^{2}$

$$
N_{3}(t+1)=N_{3}(t)+1-\frac{3 N_{3}(t)}{2 t+1}
$$

The basis for Recurrence (3) is $N_{3}(1)=4$. We prove the following lemma which shows that $\lim _{t \rightarrow+\infty} \frac{N_{3}(t)}{t}=$ $\frac{2}{5}$.

\footnotetext{
${ }^{2}$ The three initial vertices participate in one less face than their degree. However, this leaves our results unchanged.
} 
Lemma 3. $N_{3}(t)$ satisfies the following inequality:

$$
\left|N_{3}(t)-\frac{2}{5} t\right| \leq K, \text { where } K=3.6
$$

Proof. We use induction. Assume that $N_{3}(t)=\frac{2}{5} t+e_{3}(t)$, where $e_{3}(t)$ stands for the error term. We wish to prove that for all $t,\left|e_{3}(t)\right| \leq K$. The result trivially holds for $t=1$. We also see that for $t=1$ inequality (4) is tight. Assume the result holds for some $t$. We show it holds for $t+1$.

$$
\begin{aligned}
N_{3}(t+1) & =N_{3}(t)+1-\frac{3 N_{3}(t)}{2 t+1} \Rightarrow \\
e_{3}(t+1) & =e_{3}(t)+\frac{3}{5}-\frac{6 t+15 e_{3}(t)}{10 t+5}=e_{3}(t)\left(1-\frac{3}{2 t+1}\right)+\frac{3}{5(2 t+1)} \Rightarrow \\
\left|e_{3}(t+1)\right| & \leq K\left(1-\frac{3}{2 t+1}\right)+\frac{3}{5(2 t+1)} \leq K
\end{aligned}
$$

Therefore inductively Inequality (4) holds for all $t \geq 1$.

- CASE 2: $k \geq 4$ :

For $k \geq 4$ the following holds:

$$
\mathbb{E}\left[\mathbf{1}\left(d_{v}(t+1)=k\right)\right]=\mathbb{E}\left[\mathbf{1}\left(d_{v}(t)=k\right)\right]\left(1-\frac{k}{2 t+1}\right)+\mathbb{E}\left[\mathbf{1}\left(d_{v}(t)=k-1\right)\right] \frac{k-1}{2 t+1}
$$

Therefore, we can rewrite Equation (2) for $k \geq 4$ as follows:

$$
N_{k}(t+1)=N_{k}(t)\left(1-\frac{k}{2 t+1}\right)+N_{k-1}(t) \frac{k-1}{2 t+1}
$$

Lemma 4. For any $k \geq 3$, the limit $\lim _{t \rightarrow+\infty} \frac{N_{k}(t)}{t}$ exists. Specifically, let $b_{k}=\lim _{t \rightarrow+\infty} \frac{N_{k}(t)}{t}$. Then, $b_{3}=$ $\frac{2}{5}, b_{4}=\frac{1}{5}, b_{5}=\frac{4}{35}$ and for $k \geq 6 b_{k}=\frac{24}{k(k+1)(k+2)}$. Furthermore, for all $k \geq 3$

$$
\left|N_{k}(t)-b_{k} t\right| \leq K, \text { where } K=3.6 \text {. }
$$

Proof. For $k=3$ the result holds by Lemma 3 and specifically $b_{3}=\frac{2}{5}$. Assume the result holds for some $k$. We show that it holds for $k+1$ too. Rewrite Recursion (6) as: $N_{k}(t+1)=\left(1-\frac{b_{t}}{t+t_{1}}\right) N_{k}(t)+c_{t}$ where $b_{t}=$ $k / 2, t_{1}=1 / 2, c_{t}=N_{k-1}(t) \frac{k-1}{2 t+1}$. Clearly $\lim _{t \rightarrow+\infty} b_{t}=k / 2>0$ and $\lim _{t \rightarrow+\infty} c_{t}=\lim _{t \rightarrow+\infty} b_{k-1} t \frac{k-1}{2 t+1}=$ $b_{k-1}(k-1) / 2$. Hence by Lemma1

$$
\lim _{t \rightarrow+\infty} \frac{N_{k}(t)}{t}=\frac{(k-1) b_{k-1} / 2}{1+k / 2}=b_{k-1} \frac{k-1}{k+2} .
$$

Since $b_{3}=\frac{2}{5}$ we obtain that $b_{4}=\frac{1}{5}, b_{5}=\frac{4}{35}$ for any $k \geq 6, b_{k}=\frac{24}{k(k+1)(k+2)}$. This shows that the degree sequence of RANs follows a power law distribution with exponent 3 .

Now we prove Inequality (7). The case $k=3$ was proved in Lemma 3 Let $e_{k}(t)=N_{k}(t)-b_{k} t$. Assume the result holds for some $k \geq 3$, i.e., $\left|e_{k}(t)\right| \leq K$ where $K=3.6$. We show it holds for $k+1$ too. Substituting in Recurrence (2) and using the fact that $b_{k-1}(k-1)=b_{k}(k+2)$ we obtain the following:

$$
\begin{aligned}
e_{k}(t+1) & =e_{k}(t)+\frac{k-1}{2 t+1} e_{k-1}(t)-\frac{k}{2 t+1} e_{k}(t) \Rightarrow \\
\left|e_{k}(t+1)\right| & \leq\left|\left(1-\frac{k}{2 t+1}\right) e_{k}(t)\right|+\left|\frac{k-1}{2 t+1} e_{k-1}(t)\right| \leq K\left(1-\frac{1}{2 t+1}\right) \leq K
\end{aligned}
$$

Hence by induction, Inequality (7) holds for all $k \geq 3$. 
Using integration and a first moment argument, it can be seen that Lemma 4 agrees with Theorem 2 where it is shown that the maximum degree is $\approx t^{1 / 2}$. (While $b_{k}=O\left(k^{-3}\right)$ suggests a maximum degree of order $t^{1 / 3}$, summing $b_{k}$ over $k \geq K$ suggests a maximum degree of order $\left.t^{1 / 2}\right)$.

Finally, the next Lemma proves the concentration of $Z_{k}(t)$ around its expected value for $k \geq 3$. This lemma applies Lemma 2 and completes the proof of Theorem 1

Lemma 5. Let $\lambda>0$. For $k \geq 3$

$$
\operatorname{Pr}\left[\left|Z_{k}(t)-\mathbb{E}\left[Z_{k}(t)\right]\right| \geq \lambda\right] \leq e^{-\frac{\lambda^{2}}{72 t}}
$$

Proof. Let $(\Omega, \mathcal{F}, \mathbb{P})$ be the probability space induced by the construction of a RAN after $t$ insertions. Fix $k$, where $k \geq 3$, and let $\left(X_{i}\right)_{i \in\{0,1, \ldots, t\}}$ be the martingale sequence defined by $X_{i}=\mathbb{E}\left[Z_{k}(t) \mid \mathcal{F}_{i}\right]$, where $\mathcal{F}_{0}=\{\emptyset, \Omega\}$ and $\mathcal{F}_{i}$ is the $\sigma$-algebra generated by the RAN process after $i$ steps. Notice $X_{0}=$ $\mathbb{E}\left[Z_{k}(t) \mid\{\emptyset, \Omega\}\right]=N_{k}(t), X_{t}=Z_{k}(t)$. We show that $\left|X_{i+1}-X_{i}\right| \leq 6$ for $i=0, \ldots, t-1$. Let $P_{j}=\left(Y_{1}, \ldots, Y_{j-1}, Y_{j}\right), P_{j}^{\prime}=\left(Y_{1}, \ldots, Y_{j-1}, Y_{j}^{\prime}\right)$ be two sequences of face choices differing only at time $j$. Also, let $\bar{P}, \bar{P}^{\prime}$ continue from $P_{j}, P_{j}^{\prime}$ until $t$. We call the faces $Y_{j}, Y_{j}^{\prime}$ special with respect to $\bar{P}, \bar{P}^{\prime}$. We define a measure preserving map $\bar{P} \mapsto \bar{P}^{\prime}$ in the following way: for every choice of a non-special face in process $\bar{P}$ at time $l$ we make the same face choice in $\bar{P}^{\prime}$ at time $l$. For every choice of a face inside the special face $Y_{j}$ in process $\bar{P}$ we make an isomorphic (w.r.t., e.g., clockwise order and depth) choice of a face inside the special face $Y_{j}^{\prime}$ in process $\bar{P}^{\prime}$. Since the number of vertices of degree $k$ can change by at most 6, i.e., the (at most) 6 vertices involved in the two faces $Y_{j}, Y_{j}^{\prime}$ the following holds:

$$
\left|\mathbb{E}\left[Z_{k}(t) \mid P\right]-\mathbb{E}\left[Z_{k}(t) \mid P^{\prime}\right]\right| \leq 6 .
$$

Furthermore, this holds for any $P_{j}, P_{j}^{\prime}$. We deduce that $X_{i-1}$ is a weighted mean of values, whose pairwise differences are all at most 6 . Thus, the distance of the mean $X_{i-1}$ is at most 6 from each of these values. Hence, for any one step refinement $\left|X_{i+1}-X_{i}\right| \leq 6 \forall i \in\{0, \ldots, t-1\}$. By applying the Azuma-Hoeffding inequality as stated in Lemma 2 we obtain

$$
\operatorname{Pr}\left[\left|Z_{k}(t)-\mathbb{E}\left[Z_{k}(t)\right]\right| \geq \lambda\right] \leq 2 e^{-\frac{\lambda^{2}}{72 t}} .
$$

\section{Proof of Theorem 2}

We decompose the proof of Theorem 2 into several lemmas which we prove in the following. Specifically, the proof follows directly from Lemmas 7, 8, 9, 10, 11, We partition the vertices into three sets: those added before $t_{0}$, between $t_{0}$ and $t_{1}$ and after $t_{1}$ where $t_{0}=\log \log \log (f(t))$ and $t_{1}=\log \log (f(t))$. Recall that $f(t)$ is a function such that $\lim _{t \rightarrow+\infty} f(t)=+\infty$. We define a supernode to be a collection of vertices and the degree of the supernode the sum of the degrees of its vertices.

Lemma 6. Let $d_{t}(s)$ denote the degree of vertex $s$ at time $t$. and let $a^{(k)}=a(a+1) \ldots(a+k-1)$ denote the rising factorial function. Then, for any positive integer $k$

$$
\mathbb{E}\left[d_{t}(s)^{(k)}\right] \leq \frac{(k+2) !}{2}\left(\frac{2 t}{s}\right)^{\frac{k}{2}}
$$

Proof. See Appendix.

Lemma 7. The degree $X_{t}$ of the supernode $V_{t_{0}}$ of vertices added before time $t_{0}$ is at least $t_{0}^{1 / 4} \sqrt{t}$ whp.

Proof. We consider a modified process $\mathcal{Y}$ coupled with the RAN process, see also Figure 2. Specifically, let $Y_{t}$ be the modified degree of the supernode in the modified process $\mathcal{Y}$ which is defined as follows: for any type of insertion in the original RAN process -note there exist three types of insertions with respect to how the degree $X_{t}$ of the supernode (black circle) gets affected, see also Figure $2-Y_{t}$ increases by 1. We also define $X_{t_{0}}=Y_{t_{0}}$. Note that $X_{t} \geq Y_{t}$ for all $t \geq t_{0}$. Let $d_{0}=X_{t_{0}}=Y_{t_{0}}=6 t_{0}+6$ and $p^{*}=\operatorname{Pr}\left[Y_{t}=d_{0}+r \mid Y_{t_{0}}=d_{0}\right]$.

The following technical claim is proved in an appendix. 


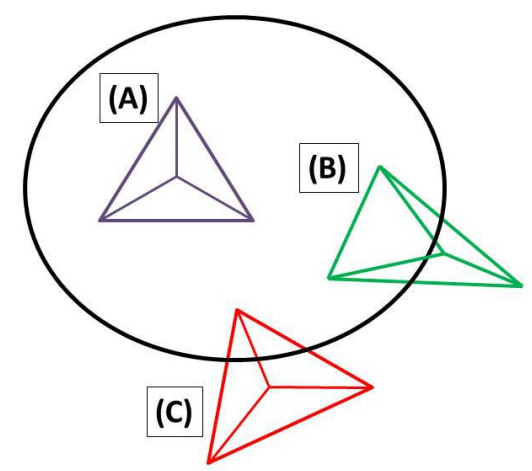

Fig. 2: Coupling used in Lemma 7

Claim (1).

$$
p^{*} \leq\left(\begin{array}{c}
d_{0}+r-1 \\
d_{0}-1
\end{array}\right)\left(\frac{2 t_{0}+3}{2 t+1}\right)^{d_{0} / 2} e^{\frac{3}{2}+t_{0}-\frac{d_{0}}{2}+\frac{2 r}{3 \sqrt{t}}}
$$

Let $\mathcal{A}_{1}$ denote the event that the supernode consisting of the first $t_{0}$ vertices has degree $Y_{t}$ in the modified process $\mathcal{Y}$ less than $t_{0}^{1 / 4} \sqrt{t}$. Note that since $\left\{X_{t} \leq t_{0}^{1 / 4} \sqrt{t}\right\} \subseteq\left\{Y_{t} \leq t_{0}^{1 / 4} \sqrt{t}\right\}$ it suffices to prove that $\operatorname{Pr}\left[Y_{t} \leq t_{0}^{1 / 4} \sqrt{t}\right]=o(1)$. Using Claim (1) we obtain

$$
\begin{aligned}
\operatorname{Pr}\left[\mathcal{A}_{1}\right] & \leq \sum_{r=0}^{t_{0}^{1 / 4} \sqrt{t}-\left(6 t_{0}+6\right)}\left(\begin{array}{c}
r+6 t_{0}+5 \\
6 t_{0}+5
\end{array}\right)\left(\frac{2 t_{0}+3}{2 t+1}\right)^{3 t_{0}+3} e^{-\frac{3}{2}-2 t_{0}+\frac{2 t_{0}^{1 / 4}}{3}} \\
& \leq t_{0}^{1 / 4} t^{1 / 2} \frac{\left(t_{0}^{1 / 4} t^{1 / 2}\right)^{6 t_{0}+5}}{\left(6 t_{0}+5\right) !}\left(\frac{2 t_{0}+3}{2 t+1}\right)^{3 t_{0}+3} e^{-\frac{3}{2}-2 t_{0}+\frac{2 t_{0}^{1 / 4}}{3}} \\
& \leq\left(\frac{t}{2 t+1}\right)^{3 t_{0}+3} \frac{t_{0}^{3 t_{0} / 2+3 / 2}\left(2 t_{0}+3\right)^{3 t_{0}+3}}{\left(6 t_{0}+5\right)^{6 t_{0}+5}} e^{4 t_{0}+7 / 2+2 / 3 t_{0}^{1 / 4}} \\
& \leq 2^{-\left(3 t_{0}+3\right)} \frac{e^{4 t_{0}+7 / 2+2 / 3 t_{0}^{1 / 4}}}{\left(6 t_{0}+5\right)^{\frac{3}{2} t_{0}+\frac{1}{2}}}=o(1) .
\end{aligned}
$$

Lemma 8. No vertex added after $t_{1}$ has degree exceeding $t_{0}^{-2} t^{1 / 2}$ whp.

Proof. Let $\mathcal{A}_{2}$ denote the event that some vertex added after $t_{1}$ has degree exceeding $t_{0}^{-2} t^{1 / 2}$. We use a union bound, a third moment argument and Lemma 6 to prove that $\operatorname{Pr}\left[\mathcal{A}_{2}\right]=o(1)$. Specifically

$$
\begin{aligned}
\operatorname{Pr}\left[\mathcal{A}_{2}\right] & \leq \sum_{s=t_{1}}^{t} \operatorname{Pr}\left[d_{t}(s) \geq t_{0}^{-2} t^{1 / 2}\right]=\sum_{s=t_{1}}^{t} \operatorname{Pr}\left[d_{t}(s)^{(3)} \geq\left(t_{0}^{-2} t^{1 / 2}\right)^{(3)}\right] \\
& \leq t_{0}^{6} t^{-3 / 2} \sum_{s=t_{1}}^{t} \mathbb{E}\left[d_{t}(s)^{(3)}\right] \leq 5 ! \sqrt{2} t_{0}^{6} \sum_{s=t_{1}}^{t} s^{-3 / 2} \leq 5 ! 2 \sqrt{2} t_{0}^{6} t_{1}^{-1 / 2}=o(1) .
\end{aligned}
$$

Lemma 9. No vertex added before $t_{1}$ has degree exceeding $t_{0}^{1 / 6} t^{1 / 2}$ whp.

Proof. Let $\mathcal{A}_{3}$ denote the event that some vertex added before $t_{1}$ has degree exceeding $t_{0}^{1 / 6} t^{1 / 2}$. We use again a third moment argument and Lemma 6 to prove that $\operatorname{Pr}\left[\mathcal{A}_{3}\right]=o(1)$. 


$$
\begin{aligned}
\operatorname{Pr}\left[\mathcal{A}_{3}\right] & \leq \sum_{s=1}^{t_{1}} \operatorname{Pr}\left[d_{t}(s) \geq t_{0}^{1 / 6} t^{1 / 2}\right]=\sum_{s=1}^{t_{1}} \operatorname{Pr}\left[d_{t}(s)^{(3)} \geq\left(t_{0}^{1 / 6} t^{1 / 2}\right)^{(3)}\right] \\
& \leq t_{0}^{-1 / 2} t^{-3 / 2} \sum_{s=1}^{t_{1}} \mathbb{E}\left[d_{t}(s)^{(3)}\right] \leq t_{0}^{-1 / 2} t^{-3 / 2} \sum_{s=1}^{t_{1}} 5 ! \sqrt{2} \frac{t^{3 / 2}}{s^{3 / 2}} \\
& \leq 5 ! \sqrt{2} \zeta(3 / 2) t_{0}^{-1 / 2}=o(1)
\end{aligned}
$$

where $\zeta(3 / 2)=\sum_{s=1}^{+\infty} s^{-3 / 2} \approx 2.612$.

Lemma 10. The $k$ highest degrees are added before $t_{1}$ and have degree $\Delta_{i}$ bounded by $t_{0}^{-1} t^{1 / 2} \leq \Delta_{i} \leq$ $t_{0}^{1 / 6} t^{1 / 2} w h p$.

Proof. For the upper bound it suffices to show that $\Delta_{1} \leq t_{0}^{1 / 6} t^{1 / 2}$. This follows immediately by Lemmas 8 and 9 . The lower bound follows directly from Lemmas 7, 8 and 9 Assume that at most $k-1$ vertices added before $t_{1}$ have degree exceeding the lower bound $t_{0}^{-1} t^{1 / 2}$. Then the total degree of the supernode formed by the first $t_{0}$ vertices is $O\left(t_{0}^{1 / 6} \sqrt{t}\right)$. This contradicts Lemma 7. Finally, since each vertex $s \geq t_{1}$ has degree at most $t_{0}^{-2} \sqrt{t} \ll t_{0}^{-1} t^{1 / 2}$ the $k$ highest degree vertices are added before $t_{1} w h p$.

The proof of Theorem 2 is completed with the following lemma whose proof is included in Appendix 8

Lemma 11. The $k$ highest degrees satisfy $\Delta_{i} \leq \Delta_{i-1}-\frac{\sqrt{t}}{f(t)}$ whp.

\section{Proof of Theorem 3}

Having computed the highest degrees of a RAN in Section 4, eigenvalues are computed by adapting existing techniques [15|22 29]. We decompose the proof of Theorem 3 in Lemmas 12, 13, 14, 15. Specifically, in Lemmas 12, 13 we bound the degrees and co-degrees respectively. Having these bounds, we decompose the graph into a star forest and show in Lemmas 14 and 15 that its largest eigenvalues, which are $(1 \pm$ $o(1)) \sqrt{\Delta_{i}}$, dominate the eigenvalues of the remaining graph. This technique was pioneered by Mihail and Papadimitriou [29].

We partition the vertices into three set $S_{1}, S_{2}, S_{3}$. Specifically, let $S_{i}$ be the set of vertices added after time $t_{i-1}$ and at or before time $t_{i}$ where

$$
t_{0}=0, t_{1}=t^{1 / 8}, t_{2}=t^{9 / 16}, t_{3}=t
$$

In the following we use the recursive variational characterization of eigenvalues [13]. Specifically, let $A_{G}$ denote the adjacency matrix of a simple, undirected graph $G$ and let $\lambda_{i}(G)$ denote the $i$-th largest eigenvalue of $A_{G}$. Then

$$
\lambda_{i}(G)=\min _{S} \max _{x \in S, x \neq 0} \frac{x^{T} A_{G} x}{x^{T} x}
$$

where $S$ ranges over all $(n-i+1)$ dimensional subspaces of $\mathbb{R}^{n}$.

Lemma 12. For any $\epsilon>0$ and any $f(t)$ with $f(t) \rightarrow+\infty$ as $t \rightarrow+\infty$ the following holds whp: for all $s$ with $f(t) \leq s \leq t$, for all vertices $r \leq s$, then $d_{s}(r) \leq s^{\frac{1}{2}+\epsilon} r^{-\frac{1}{2}}$.

Proof. Set $q=\left\lceil\frac{4}{\epsilon}\right\rceil$. We use Lemma 6, a union bound and Markov's inequality to obtain: 


$$
\begin{aligned}
\operatorname{Pr}\left[\bigcup_{s=f(t)}^{t} \bigcup_{r=1}^{s}\left\{d_{s}(r) \geq s^{1 / 2+\epsilon} r^{-1 / 2}\right\}\right] & \leq \sum_{s=f(t)}^{t} \sum_{r=1}^{s} \operatorname{Pr}\left[d_{s}(r)^{(q)} \geq\left(s^{1 / 2+\epsilon} r^{-1 / 2}\right)^{(q)}\right] \\
& \leq \sum_{s=f(t)}^{t} \sum_{r=1}^{s} \operatorname{Pr}\left[d_{s}(r)^{(q)} \geq\left(s^{-(q / 2+q \epsilon)} r^{q / 2}\right)\right] \\
& \leq \sum_{s=f(t)}^{t} \sum_{r=1}^{s} \frac{(q+2) !}{2}\left(\frac{2 s}{r}\right)^{q / 2} s^{-q / 2} s^{-q \epsilon} r^{q / 2} \\
& =\frac{(q+2) !}{2} 2^{q / 2} \sum_{s=f(t)}^{t} s^{1-q \epsilon} \\
& \leq \frac{(q+2) !}{2} 2^{q / 2} \int_{f(t)-1}^{t} x^{1-q \epsilon} \mathrm{d} x \\
& \leq \frac{(q+2) !}{2(q \epsilon-2)} 2^{q / 2}(f(t)-1)^{2-q \epsilon}=o(1) .
\end{aligned}
$$

Lemma 13. Let $S_{3}^{\prime}$ be the set of vertices in $S_{3}$ which are adjacent to more than one vertex of $S_{1}$. Then $\left|S_{3}^{\prime}\right| \leq t^{1 / 6}$ whp.

Proof. First, observe that when vertex $s$ is inserted it becomes adjacent to more than one vertex of $S_{1}$ if the face chosen by $s$ has at least two vertices in $S_{1}$. We call the latter property $\mathcal{A}$ and we write $s \in \mathcal{A}$ when $s$ satisfies it. At time $t_{1}$ there exist $2 t_{1}+1$ faces total, which consist of faces whose three vertices are all from $S_{1}$. At time $s \geq t_{2}$ there can be at most $6 t_{1}+3$ faces with at least two vertices in $S_{1}$ since each of the original $2 t_{1}+1$ faces can give rise to at most 3 new faces with at least two vertices in $s_{1}$. Consider a vertex $s \in S_{3}$, i.e., $s \geq t_{2}$. By the above argument, $\operatorname{Pr}\left[\left|N(s) \cap S_{1}\right| \geq 2\right] \leq \frac{6 t_{1}+3}{2 t+1}$. Writing $\left|S_{3}^{\prime}\right|$ as a sum of indicator variables, i.e., $\left|S_{3}^{\prime}\right|=\sum_{s=t_{2}}^{t} I(s \in \mathcal{A})$ and taking the expectation we obtain

$$
\begin{aligned}
\mathbb{E}\left[\left|S_{3}^{\prime}\right|\right] & \leq \sum_{s=t_{2}}^{t} \frac{6 t_{1}+3}{2 t+1} \leq\left(6 t_{1}+3\right) \int_{t_{2}}^{t}(2 x+1)^{-1} \mathrm{~d} x \\
& \leq\left(3 t^{\frac{1}{8}}+\frac{3}{2}\right) \ln \frac{2 t+1}{2 t_{2}+1}=o\left(t^{1 / 7}\right)
\end{aligned}
$$

By Markov’s inequality:

$$
\operatorname{Pr}\left[\left|S_{3}^{\prime}\right| \geq t^{1 / 6}\right] \leq \frac{\mathbb{E}\left[\left|S_{3}^{\prime}\right|\right]}{t^{1 / 6}}=o(1) .
$$

Therefore, we conclude that $\left|S_{3}^{\prime}\right| \leq t^{1 / 6}$ whp.

Lemma 14. Let $F \subseteq G$ be the star forest consisting of edges between $S_{1}$ and $S_{3}-S_{3}^{\prime}$. Let $\Delta_{1} \geq \Delta_{2} \geq$ $\ldots \geq \Delta_{k}$ denote the $k$ highest degrees of $G$. Then $\lambda_{i}(F)=(1-o(1)) \sqrt{\Delta_{i}}$ whp.

Proof. It suffices to show that $\Delta_{i}(F)=(1-o(1)) \Delta_{i}(G)$ for $i=1, \ldots, k$. Note that since the $k$ highest vertices are inserted before $t_{1} w h p$, the edges they lose are the edges between $S_{1}$ and the ones incident to $S_{3}^{\prime}$ and $S_{2}$ and we know how to bound the cardinalities of all these sets. Specifically by Lemma $13\left|S_{3}^{\prime}\right| \leq t^{1 / 6}$ whp and by Theorem 2 the maximum degree in $G_{t_{1}}, G_{t_{2}}$ is less than $t_{1}^{1 / 2+\epsilon_{1}}=t^{1 / 8}, t_{2}^{1 / 2+\epsilon_{2}}=t^{5 / 16}$ for $\epsilon_{1}=1 / 16, \epsilon_{2}=1 / 32$ respectively $w h p$. Also by Theorem $2, \Delta_{i}(G) \geq \frac{\sqrt{t}}{\log t}$. Hence, we obtain

$$
\Delta_{i}(F) \geq \Delta_{i}(G)-t^{1 / 8}-t^{5 / 16}-t^{1 / 6}=(1-o(1)) \Delta_{i}(G) .
$$


To complete the proof of Theorem 3 it suffices to prove that $\lambda_{1}(H)$ is $o\left(\lambda_{k}(F)\right)$ where $H=G-F$. We prove this in the following lemma. The proof is based on bounding maximum degree of appropriately defined subgraphs using Lemma 12 and standard inequalities from spectral graph theory [13].

Lemma 15. $\lambda_{1}(H)=o\left(t^{1 / 4}\right) w h p$.

Proof. From Gershgorin's theorem [32] the maximum eigenvalue of any graph is bounded by the maximum degree. We bound the eigenvalues of $H$ by bounding the maximum eigenvalues of six different induced subgraphs. Specifically, let $H_{i}=H\left[S_{i}\right], H_{i j}=H\left(S_{i}, S_{j}\right)$ where $H[S]$ is the subgraph induced by the vertex set $S$ and $H(S, T)$ is the subgraph containing only edges with one vertex is $S$ and other in $T$. We use Lemma 14 to bound $\lambda_{1}\left(H\left(S_{1}, S_{3}\right)\right)$ and Lemma 13 for the other eigenvalues. We set $\epsilon=1 / 64$.

$$
\begin{gathered}
\lambda_{1}\left(H_{1}\right) \leq \Delta_{1}\left(H_{1}\right) \leq t_{1}^{1 / 2+\epsilon}=t^{33 / 512} . \\
\lambda_{1}\left(H_{2}\right) \leq \Delta_{1}\left(H_{2}\right) \leq t_{2}^{1 / 2+\epsilon} t_{1}^{-1 / 2}=t^{233 / 1024} . \\
\lambda_{1}\left(H_{3}\right) \leq \Delta_{1}\left(H_{3}\right) \leq t_{3}^{1 / 2+\epsilon} t_{2}^{-1 / 2}=t^{15 / 64} . \\
\lambda_{1}\left(H_{12}\right) \leq \Delta_{1}\left(H_{12}\right) \leq t_{2}^{1 / 2+\epsilon}=t^{297 / 1024} . \\
\lambda_{1}\left(H_{23}\right) \leq \Delta_{1}\left(H_{23}\right) \leq t_{3}^{1 / 2+\epsilon} t_{1}^{-1 / 2}=t^{29 / 64} . \\
\lambda_{1}\left(H_{13}\right) \leq \Delta_{1}\left(H_{13}\right) \leq t^{1 / 6} .
\end{gathered}
$$

Therefore $w h p$ we obtain

$$
\lambda_{1}(H) \leq \sum_{i=1}^{3} \lambda_{1}\left(H_{i}\right)+\sum_{i<j} \lambda_{1}\left(H_{i, j}\right)=o\left(t^{1 / 4}\right) .
$$

\section{Proof of Theorem 4}

Before we give the proof of Theorem 4 , we give a simple proof that the diameter of a RAN is $O(\log t) w h p$.

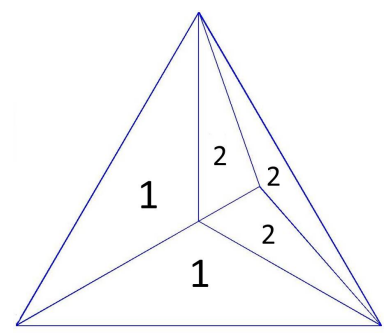

Fig. 3: An instance of the process for $t=2$. Each face is labeled with its depth.

We begin with a necessary definition for the proof of Claim (2). We define the depth of a face recursively. Initially, we have three faces, see Figure 1 a, whose depth equals 1 . For each new face $\beta$ created by picking a face $\gamma$, we have $\operatorname{depth}(\beta)=\operatorname{depth}(\gamma)+1$. An example is shown in Figure 3 , where each face is labeled with its corresponding depth.

Claim (2). The diameter $d\left(G_{t}\right)$ satisfies $d\left(G_{t}\right)=O(\log t)$ whp. 
Proof. A simple but key observation is that if $k^{*}$ is the maximum depth of a face then $d\left(G_{t}\right)=O\left(k^{*}\right)$. Hence, we need to upper bound the depth of a given face after $t$ rounds. Let $F_{t}(k)$ be the number of faces of depth $k$ at time $t$, then:

$$
\mathbb{E}\left[F_{t}(k)\right]=\sum_{1 \leq t_{1}<t_{2}<\ldots<t_{k} \leq t} \prod_{j=1}^{k} \frac{1}{2 t_{j}+1} \leq \frac{1}{k !}\left(\sum_{j=1}^{t} \frac{1}{2 j+1}\right)^{k} \leq \frac{1}{k !}\left(\frac{1}{2} \log t\right)^{k} \leq\left(\frac{e \log t}{2 k}\right)^{k+1}
$$

By the first moment method we obtain $k^{*}=O(\log t)$ whp and by our observation $d\left(G_{t}\right)=O(\log t)$ whp.

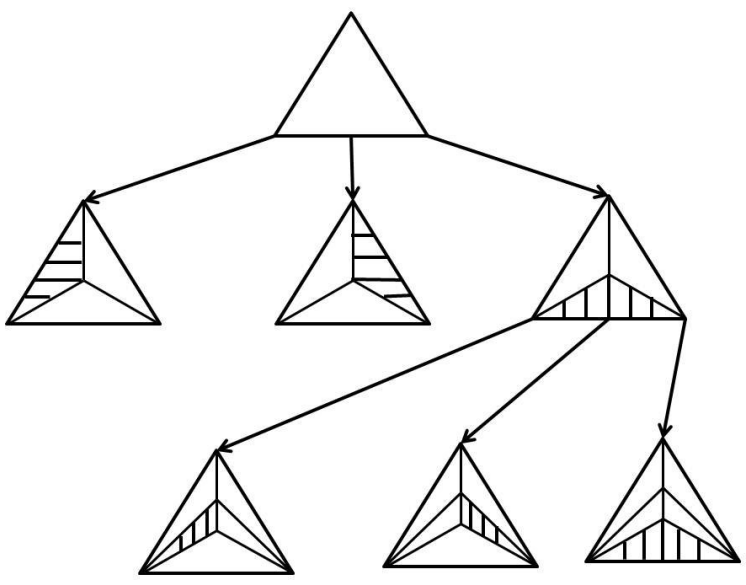

Fig. 4: RANs as random ternary trees.

The depth of a face can be formalized via a bijection between random ternary trees and RANs. Using this bijection we prove Theorem 4 which gives a refined upper bound on the asymptotic growth of the diameter.

Proof. Consider the random process which starts with a single vertex tree and at every step picks a random leaf and adds three children to it. Let $T$ be the resulting tree after $t$ steps. There exists a natural bijection between the RAN process and this process, see [18] and also Figure 4 . The depth of $T$ in probability is $\frac{\rho}{2} \log t$ where $\frac{1}{\rho}=\eta$ is the unique solution greater than 1 of the equation $\eta-1-\log \eta=\log 3$, see Broutin and Devroye [12], pp. 284-285. Note that the diameter $d\left(G_{t}\right)$ is at most twice the height of the tree and hence the result follows.

The above observation, i.e., the bijection between RANs and random ternary trees cannot be used to lower bound the diameter. A counterexample is shown in Figure 5 where the height of the random ternary tree can be made arbitrarily large but the diameter is 2. Albenque and Marckert proved in [2] that if $v, u$ are two i.i.d. uniformly random internal vertices, i.e., $v, u \geq 4$, then the distance $d(u, v)$ tends to $\frac{6}{11} \log n$ with probability 1 as the number of vertices $n$ of the RAN grows to infinity. However, an exact expression of the asymptotic growth of the diameter to the best of our knowledge remains an open problem. Finally, it is worth mentioning that the diameter of the RAN grows faster asymptotically than the diameter of the classic preferential attachment model [7] which whp grows as $\frac{\log t}{\log \log t}$, see Bollobás and Riordan [9].

\section{Waiting Times}

Consider the three initial faces of Figure 1a. Let's call the face which receives the first vertex $A$ and the other two faces $B, C$. Let $X$ equal the number of steps until a new vertex picks face $B$ or $C$. Clearly, $X \in\{1,2, \ldots\}$. What is the expectation $\mathbb{E}[X]$ ? For any $t \geq 1$ 

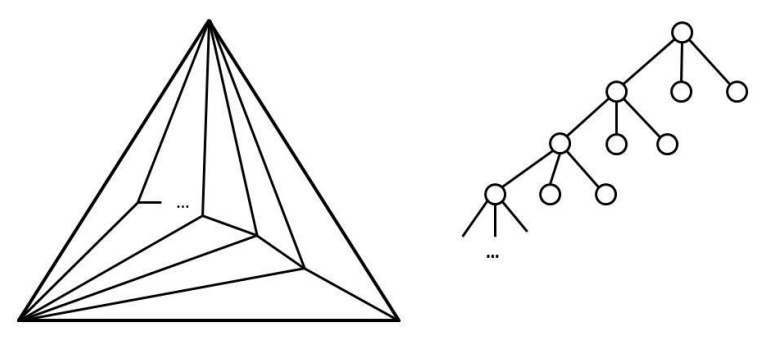

Fig. 5: The height of the random ternary tree cannot be used to lower bound the diameter. The height of the random ternary tree can be arbitrarily large but the diameter is 2 .

$$
\operatorname{Pr}[X>t]=\prod_{j=1}^{t} \frac{3+2(j-1)}{5+2(j-1)}=\frac{3}{2 t+3}
$$

Using now the fact that $\mathbb{E}[X]=\sum_{t=1}^{+\infty} \operatorname{Pr}[X \geq t]=1+\sum_{t=1}^{+\infty} \operatorname{Pr}[X>t]$ we obtain that $\mathbb{E}[X]=+\infty$.

\section{Open Problems}

We propose three open problems for future work. The first concerns the diameter. Specifically, as we mentioned also earlier, an interesting problem is to find an exact asymptotic expression for the diameter of RAN.

Conductance: We conjecture that the conductance of a RAN is $\Theta\left(\frac{1}{\sqrt{t}}\right)$ whp. Figure 6 shows that $\Phi\left(G_{t}\right) \leq$ $\frac{1}{\sqrt{t}}$.

Hamiltonicity and Longest Path: We conjecture that whp a RAN is not Hamiltonian but the length of the longest path is $\Omega(n)$.

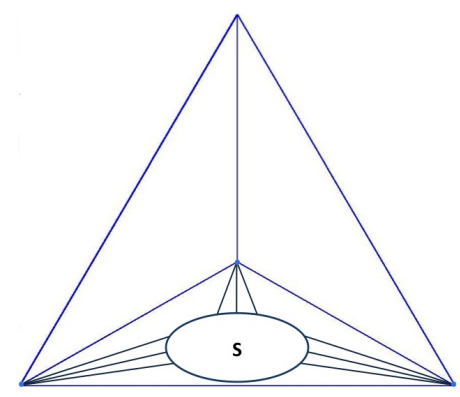

Fig. 6: By the pigeonhole principle, one of the three initial faces receives $\Theta(t)$ vertices. Using Theorem 2 it is not hard to see that the encircled set of vertices $S$ has conductance $\phi(S) \approx \frac{\sqrt{t}}{t}=\frac{1}{\sqrt{t}} w h p$.

\section{Acknowledgements}

Research supported by NSF Grant No. CCF-1013110. We would like to thank Luc Devroye and Alexis Darrasse for pointing out references [12] and [2]30] respectively. 


\section{References}

1. Aiello, W., Chung, F., Lu, L.: A random graph model for power law graphs Experimental Mathemathics, 10(1), 53-66, 2001

2. Albenque, M., Marckert, J.F.: Some families of increasing planar maps Electronic Journal of Probability, 13, pp. 1624-1671

3. Alon, N., Spencer, J.: The Probabilistic Method. Wiley-Interscience (2008)

4. Andrade, J.S., Herrmann, H.J., Andrade, R.F.S., da Silva, L.R.: Apollonian networks: simultaneously scale-free, small world Euclidean, space filling, and with matching graphs Phys. Rev. Lett. 94 (2005) 018702.

5. Andrade, R.F.S., Miranda, J.G.V.: Spectral Properties of the Apollonian Network Physica A, 356, (2005)

6. Azuma, K.: Weighted sums of certain dependent variables Tohoku Math J 3, pp. 357-367, 1967

7. Barabási, A., Albert, R.: Emergence of Scaling in Random Networks Science 286, pp. 509512, 1999

8. Bodini, O., Darrasse, A., Soria, M.: Distances in random Apollonian network structures Available at Arxiv http: //arxiv.org/abs/0712.2129

9. Bollobás, B., Riordan, O.: The Diameter of a Scale-Free Random Graph Combinatorica, 2002

10. Bollobás, B., Riordan, O., Spencer, J., Tusnády, G.: The Degree Sequence of a Scale Free Random Graph Process Random Struct. Algorithms, 18(3), pp. 279-290, 2001

11. Boyd, D.W.: The Sequence of Radii of the Apollonian Packing Mathematics of Computation, Vol. 19, pp. 249-254, 1982

12. Broutin, N., Devroye, L.: Large Deviations for the Weighted Height of an Extended Class of Trees Algorithmica, 46, pp. 271-297, 2006

13. Chung Graham, F.: Spectral Graph Theory American Mathematical Society 1997

14. Chung Graham, F., Lu, L.: Complex Graphs and Networks American Mathematical Society, No. 107 (2006)

15. Chung, F., Lu, L, Vu. V.H.: Spectra of random graphs with given expected degrees Proceedings of the National Academy of Sciences of the United States of America, 100, 6313-6318

16. Cooper, C., Frieze, A.: A general model of web graphs Random Structures \& Algorithms, Volume 22 Issue 3,pp. 311-335, 2003

17. Cooper, C., Uehara, R.: Scale Free Properties of random k-trees Mathematics in Computer Science, 3(4), pp. 489-496, 2010

18. Darrasse, A., Soria, M.: Degree distribution of random Apollonian network structures and Boltzmann sampling 2007 Conference on Analysis of Algorithms, AofA 07, DMTCS Proceedings.

19. Darrasse, A., Hwang, H.-K., Bodini, O., Soria, M.: The connectivity-profile of random increasing k-trees Available at Arxiv: http://arxiv.org/abs/0910.3639

20. Doye, J.P.K, Massen, C.P.: Self-similar disk packings as model spatial scale-free networks Phys. Rev. E 71 (2005) 016128.

21. Fabrikant, A., Koutsoupias, E., Papadimitriou, C.: Heuristically Optimized Trade-offs Proceedings of the 29th International Colloquium on Automata, Languages, and Programming (ICALP), pp. 110-122, 2002

22. Flaxman, A., Frieze, A., Fenner, T.: High Degree Vertices and Eigenvalues in the Preferential Attachment Graph Internet Mathematics, 2(1), 2005

23. Gao, Y.: The degree distribution of random k-trees Theoretical Computer Science, 410(8-10), 2009.

24. Gao, Y., Hobson, C.: . Random k-tree as a model for complex networks Workshop on Algorithms and Models for the Web-Graph (WAW), 2006

25. Graham, R.L., Lagarias, J.C., Mallows, C.L., Wilks, A.R., Yan, C.H.: Apollonian Circle Packings: Number Theory J. Number Theory 100, No. 1, 1-45, MR1971245

26. Hoeffding, W.: Probability inequalities for sumes of bounded random variables J Amer Statist Assoc 58, pp. 13-30, 1963

27. Kloks, T.: Treewidth: Computations and Approximations Springer-Verlag, 1994

28. Leskovec, J., Faloutsos, C.: Scalable modeling of real graphs using Kronecker multiplication Machine Learning Proceedings of the Twenty-Fourth International Conference (ICML 2007), Corvalis, Oregon, USA, June 20-24, 2007

29. Mihail, M., Papadimitriou, C.: On the Eigenvalue Power Law RANDOM '02, pp. 254-262 (2002)

30. Panholzer, A., Seitz, G.: Ordered increasing k-trees: Introduction and analysis of a preferential attachment network model DMTCS proc., AofA'10, pp. 549-564, 2010

31. Pralat, P., Wormald, N.: Growing Protean Graphs Internet Mathematics, 4(1), pp. 1-16, 2007

32. Strang, G.: Linear Algebra and Its Applications Brooks Cole, 2005

33. Wu, Z.-X., Xu, X.-J., Wang, Y.-H.: Comment on "Maximal planar networks with large clustering coefficient and power-law degree distribution” Physical Review, E 73, 058101 (2006)

34. Zhang, Z.Z., Comellas, F., Fertin, G., Rong, L.L.: High dimensional Apollonian networks Available at ArXiv http://arxiv.org/abs/cond-mat/0503316

35. Zhou, T., Yan, G., Wang, B.H.: Maximal planar networks with large clustering coefficient and power-law degree distribution Phys. Rev. E 71 (2005) 046141. 


\section{Appendix}

\section{Proof of Lemma 6}

Proof. As we mentioned in the proof of Theorem 1 the three initial vertices $1,2,3$ have one less face than their degree whereas all other vertices have degree equal to the number of faces surrounding them. In this proof we treat both cases but we omit it in all other proofs.

- CASE 1:s $\geq 4$

Note that $d_{s}(s)=3$. By conditioning successively we obtain

$$
\begin{aligned}
\mathbb{E}\left[d_{t}(s)^{(k)}\right] & =\mathbb{E}\left[\mathbb{E}\left[d_{t}(s)^{(k)} \mid d_{t-1}(s)\right]\right] \\
& =\mathbb{E}\left[\left(d_{t-1}(s)\right)^{(k)}\left(1-\frac{d_{t-1}(s)}{2 t-1}\right)+\left(d_{t-1}(s)+1\right)^{(k)} \frac{d_{t-1}(s)}{2 t-1}\right] \\
& =\mathbb{E}\left[\left(d_{t-1}(s)\right)^{(k)}\left(1-\frac{d_{t-1}(s)}{2 t-1}\right)+\left(d_{t-1}(s)\right)^{(k)} \frac{d_{t-1}(s)+k}{d_{t-1}(s)} \frac{d_{t-1}(s)}{2 t-1}\right] \\
& =\mathbb{E}\left[\left(d_{t-1}(s)\right)^{(k)}\right]\left(1+\frac{k}{2 t-1}\right)=\ldots=3^{(k)} \prod_{t^{\prime}=s+1}^{t}\left(1+\frac{k}{2 t^{\prime}-1}\right) \\
& \leq 3^{(k)} \exp \left(\sum_{t^{\prime}=s+1}^{t} \frac{k}{2 t^{\prime}-1}\right) \leq 3^{(k)} \exp \left(k \int_{s}^{t} \frac{\mathrm{d} x}{2 x-1}\right) \\
& \leq \frac{(k+2) !}{2} \exp \left(\frac{k}{2} \log \frac{t-1 / 2}{s-1 / 2}\right) \leq \frac{(k+2) !}{2}\left(\frac{2 t}{s}\right)^{\frac{k}{2}} .
\end{aligned}
$$

- CASE 2: $s \in\{1,2,3\}$

Note that initially the degree of any such vertex is 2 . For any $k \geq 0$

$$
\begin{aligned}
\mathbb{E}\left[d_{t}(s)^{(k)}\right] & =\mathbb{E}\left[\mathbb{E}\left[d_{t}(s)^{(k)} \mid d_{t-1}(s)\right]\right] \\
& =\mathbb{E}\left[\left(d_{t-1}(s)\right)^{(k)}\left(1-\frac{d_{t-1}(s)-1}{2 t-1}\right)+\left(d_{t-1}(s)+1\right)^{(k)} \frac{d_{t-1}(s)-1}{2 t-1}\right] \\
& =\mathbb{E}\left[\left(d_{t-1}(s)\right)^{(k)}\left(1+\frac{k}{2 t-1}\right)-\left(d_{t-1}(s)\right)^{(k)} \frac{k}{(2 t-1) d_{t-1}(s)}\right] \\
& \leq \mathbb{E}\left[\left(d_{t-1}(s)\right)^{(k)}\right]\left(1+\frac{k}{2 t-1}\right) \leq \ldots \leq \frac{(k+2) !}{2}\left(\frac{2 t}{s}\right)^{\frac{k}{2}} .
\end{aligned}
$$

\section{Proof of Claim (1)}

Proof. Let $\tau=(t_{0} \equiv \tau_{0}, \underbrace{\tau_{1}, \ldots, \tau_{r}}_{\text {insertion times }}, \tau_{r+1} \equiv t)$ be a vector denoting that $Y_{t}$ increases by 1 at $\tau_{i}$ for $i=1, \ldots, r$. We upper bound the probability $p_{\tau}$ of this event in the following. Note that we consider the case where the vertices have same degree as the number of faces around them. As we mentioned earlier, the other case is analyzed in exactly the same way, modulo a negligible error term. 


$$
\begin{aligned}
p_{\tau} & =\left[\prod_{k=1}^{r} \frac{d_{0}+k-1}{2 \tau_{k}+1}\right]\left[\prod_{k=0}^{r} \prod_{j=\tau_{k}+1}^{\tau_{k+1}-1}\left(1-\frac{d_{0}+k}{2 j+1}\right)\right] \\
& =d_{0}\left(d_{0}+1\right) \ldots\left(d_{0}+r-1\right)\left[\prod_{k=1}^{r} \frac{1}{2 \tau_{k}+1}\right] \exp \left(\sum_{k=0}^{r} \sum_{j=\tau_{k}+1}^{\tau_{k+1}-1} \log \left(1-\frac{d_{0}+k}{2 j+1}\right)\right) \\
& =\frac{\left(d_{0}+r-1\right) !}{\left(d_{0}-1\right) !}\left[\prod_{k=1}^{r} \frac{1}{2 \tau_{k}+1}\right] \exp \left(\sum_{k=0}^{r} \sum_{j=\tau_{k}+1}^{\tau_{k+1}-1} \log \left(1-\frac{d_{0}+k}{2 j+1}\right)\right)
\end{aligned}
$$

Consider now the inner sum which we upper bound using an integral:

$$
\begin{aligned}
\sum_{j=\tau_{k}+1}^{\tau_{k+1}-1} \log \left(1-\frac{d_{0}+k}{2 j+1}\right) & \leq \int_{\tau_{k}+1}^{\tau_{k+1}} \log \left(1-\frac{d_{0}+k}{2 x+1}\right) \mathrm{d} x \\
& \leq-\left(\tau_{k+1}+\frac{1}{2}\right) \log \left(2 \tau_{k+1}+1\right)+ \\
& \frac{2 \tau_{k+1}+1-\left(d_{0}+k\right)}{2} \log \left(2 \tau_{k+1}+1-\left(d_{0}+k\right)\right)+ \\
& \left(\tau_{k}+\frac{3}{2}\right) \log \left(2 \tau_{k}+3\right)-\frac{2 \tau_{k}+3-\left(d_{0}+k\right)}{2} \log \left(2 \tau_{k}+3-\left(d_{0}+k\right)\right)
\end{aligned}
$$

since

$$
\int \log \left(1-\frac{d_{0}+k}{2 x+1}\right)=-\left(x+\frac{1}{2}\right) \log (2 x+1)+\frac{2 x+1-\left(d_{0}+k\right)}{2} \log \left(2 x+1-\left(d_{0}+k\right)\right)
$$

Hence we obtain $\sum_{k=0}^{r} \sum_{j=\tau_{k}+1}^{\tau_{k+1}-1} \log \left(1-\frac{d_{0}+k}{2 j+1}\right) \leq A+\sum_{k=1}^{r} B_{k}$ where

$$
\begin{aligned}
A & =\left(\tau_{0}+\frac{3}{2}\right) \log \left(2 \tau_{0}+3\right)-\frac{2 \tau_{0}+3-d_{0}}{2} \log \left(2 \tau_{0}+3-d_{0}\right) \\
& -\left(\tau_{r+1}+\frac{1}{2}\right) \log \left(2 \tau_{r+1}+1\right)+\frac{2 \tau_{r+1}+1-\left(d_{0}+r\right)}{2} \log \left(2 \tau_{r+1}+1-\left(d_{0}+r\right)\right)
\end{aligned}
$$

and

$$
\begin{aligned}
B_{k} & =\left(\tau_{k}+\frac{3}{2}\right) \log \left(2 \tau_{k}+3\right)-\frac{2 \tau_{k}+3-\left(d_{0}+k\right)}{2} \log \left(2 \tau_{k}+3-\left(d_{0}+k\right)\right) \\
& -\left(\tau_{k}+\frac{1}{2}\right) \log \left(2 \tau_{k}+1\right)+\frac{2 \tau_{k}+1-\left(d_{0}+k-1\right)}{2} \log \left(2 \tau_{k}+1-\left(d_{0}+k-1\right)\right) .
\end{aligned}
$$

We first upper bound the quantities $B_{k}$ for $k=1, \ldots, r$. By rearranging terms and using the identity $\log (1+x) \leq x$ we obtain

$$
\begin{aligned}
B_{k} & =\left(\tau_{k}+\frac{1}{2}\right) \log \left(1+\frac{1}{\tau_{k}+\frac{1}{2}}\right)+\log \left(2 \tau_{k}+3\right) \\
& -\frac{1}{2} \log \left(2 \tau_{k}+3-\left(d_{0}+k\right)\right)-\frac{2 \tau_{k}+2-\left(d_{0}+k\right)}{2} \log \left(1+\frac{1}{2 \tau_{k}+2-\left(d_{0}+k\right)}\right) . \\
& \leq \frac{1}{2}+\frac{1}{2} \log \left(2 \tau_{k}+3\right)-\frac{1}{2} \log \left(1-\frac{d_{0}+k}{2 \tau_{k}+3}\right)
\end{aligned}
$$

First we rearrange terms and then we bound the term $e^{A}$ by using the inequality $e^{-x-x^{2} / 2} \geq 1-x$ which is valid for $0<x<1$ : 


$$
\begin{aligned}
A & =-\left(\tau_{0}+\frac{3}{2}\right) \log \left(1-\frac{d_{0}}{2 \tau_{0}+3}\right)+\left(\tau_{r+1}+\frac{1}{2}\right) \log \left(1-\frac{d_{0}+r}{2 \tau_{r+1}+1}\right)+\frac{d_{0}}{2} \log \left(2 \tau_{0}+3-d_{0}\right) \\
& -\frac{d_{0}+r}{2} \log \left(2 \tau_{r+1}+1-\left(d_{0}+r\right)\right) . \Rightarrow \\
e^{A} & =\left(1-\frac{d_{0}}{2 \tau_{0}+3}\right)^{-\left(\tau_{0}+\frac{3}{2}\right)}\left(1-\frac{d_{0}+r}{2 \tau_{r+1}+1}\right)^{\tau_{r+1}+\frac{1}{2}}\left(2 \tau_{0}+3-d_{0}\right)^{\frac{d_{0}}{2}}\left(2 \tau_{r+1}+1-\left(d_{0}+r\right)\right)^{-\frac{d_{0}+r}{2}} \\
& =\left(\frac{2 \tau_{0}+3}{2 \tau_{r+1}+1}\right)^{d_{0} / 2}\left(2 \tau_{r+1}+1\right)^{-r / 2}\left(1-\frac{d_{0}}{2 \tau_{0}+3}\right)^{-\left(\tau_{0}+\frac{3}{2}\right)+\frac{d_{0}}{2}}\left(1-\frac{d_{0}+r}{2 \tau_{r+1}+1}\right)^{\tau_{r+1}+\frac{1}{2}-\frac{d_{0}+r}{2}} \\
& \leq\left(\frac{2 t_{0}+3}{2 t+1}\right)^{d_{0} / 2}(2 t+1)^{-r / 2}\left(1-\frac{d_{0}}{2 \tau_{0}+3}\right)^{-\left(\tau_{0}+\frac{3}{2}\right)+\frac{d_{0}}{2}} e^{\left(-\frac{d_{0}+r}{2 t+1}-\left(-\frac{d_{0}+r}{2 t+1}\right)^{2} / 2\right)\left(t+1 / 2-\frac{d_{0}+r}{2}\right)} \\
& =\left(\frac{2 t_{0}+3}{2 t+1}\right)^{d_{0} / 2}(2 t+1)^{-r / 2}\left(1-\frac{d_{0}}{2 \tau_{0}+3}\right)^{-\left(\tau_{0}+\frac{3}{2}\right)+\frac{d_{0}}{2}} e^{-\frac{d_{0}+r}{2}+\frac{\left(d_{0}+r\right)^{2}}{8 t+4}+\frac{\left(d_{0}+r\right)^{3}}{4(2 t+1)^{2}}}
\end{aligned}
$$

Now we upper bound the term $\exp \left(A+\sum_{k=1}^{r} B_{k}\right)$ using the above upper bounds:

$$
\begin{aligned}
e^{A+\sum_{k=1}^{r} B_{k}} & \leq e^{A} e^{r / 2} \prod_{i=1}^{r} \sqrt{\frac{2 \tau_{k}+3}{1-\frac{d_{0}+k}{2 \tau_{k}+3}}} \\
& \leq\left(1-\frac{d_{0}}{2 \tau_{0}+3}\right)^{-\left(\tau_{0}+\frac{3}{2}\right)+\frac{d_{0}}{2}} e^{-\frac{d_{0}}{2}+\frac{\left(d_{0}+r\right)^{2}}{8 t+4}+\frac{\left(d_{0}+r\right)^{3}}{4(2 t+1)^{2}}\left(\frac{2 t_{0}+3}{2 t+1}\right)^{d_{0} / 2} \times} \\
& (2 t+1)^{-r / 2} \prod_{i=1}^{r} \sqrt{\frac{2 \tau_{k}+3}{1-\frac{d_{0}+k}{2 \tau_{k}+3}}}
\end{aligned}
$$

Using the above upper bound we get that

$$
p_{\tau} \leq C\left(r, d_{0}, t_{0}, t\right) \prod_{k=1}^{r}\left[\left(2 \tau_{k}+3-\left(d_{0}+k\right)\right)^{-1 / 2}\left(1+\frac{1}{\tau_{k}+1 / 2}\right)\right]
$$

where

$C\left(r, d_{0}, t_{0}, t\right)=\frac{\left(d_{0}+r-1\right) !}{\left(d_{0}-1\right) !}\left(1-\frac{d_{0}}{2 \tau_{0}+3}\right)^{-\left(\tau_{0}+\frac{3}{2}\right)+\frac{d_{0}}{2}} e^{-\frac{d_{0}}{2}+\frac{\left(d_{0}+r\right)^{2}}{8 t+4}+\frac{\left(d_{0}+r\right)^{3}}{4(2 t+1)^{2}}}\left(\frac{2 t_{0}+3}{2 t+1}\right)^{d_{0} / 2}(2 t+1)^{-r / 2}$

We need to sum over all possible insertion times to bound the probability of interest $p^{*}$. We set $\tau_{k}^{\prime} \leftarrow$ $\tau_{k}-\left\lceil\frac{d_{0}+k}{2}\right\rceil$ for $k=1, \ldots, r$. For $d=o(\sqrt{t})$ and $r=o\left(t^{2 / 3}\right)$ we obtain: 


$$
\begin{aligned}
p^{*} & \leq C\left(r, d_{0}, t_{0}, t\right) \sum_{t_{0}+1 \leq \tau_{1}<. .<\tau_{r} \leq t} \prod_{k=1}^{r}\left[\left(2 \tau_{k}+3-\left(d_{0}+k\right)\right)^{-1 / 2}\left(1+\frac{1}{\tau_{k}+1 / 2}\right)\right] \\
& \leq C\left(r, d_{0}, t_{0}, t\right) \quad \sum_{t_{0}-\left\lceil\frac{d_{0}}{2}\right\rceil+1 \leq \tau_{1}^{\prime} \leq . . \leq \tau_{r}^{\prime} \leq t-\left\lceil\frac{d_{0}+r}{2}\right\rceil^{\prime}}^{r}\left[\left(2 \tau_{k}^{\prime}+3\right)^{-1 / 2}\left(1+\frac{1}{\tau_{k}^{\prime}+\frac{d_{0}+k}{2}+1 / 2}\right)\right] \\
& \leq \frac{C\left(r, d_{0}, t_{0}, t\right)}{r !}\left(\sum_{t_{0}-\left\lceil\frac{d_{0}}{2}\right\rceil}^{t-\left\lceil\frac{d_{0}+r}{2}\right\rceil}\left(2 \tau_{k}^{\prime}+3\right)^{-1 / 2}+\frac{1}{\sqrt{2}}\left(\tau_{k}^{\prime}+3 / 2\right)^{-3 / 2}\right)^{r} \\
& \leq \frac{C\left(r, d_{0}, t_{0}, t\right)}{r !}\left(\int_{0}^{t-\frac{d+r}{2}}\left[(2 x+3)^{-1 / 2}+\frac{1}{\sqrt{2}}(x+3 / 2)^{-3 / 2}\right] \mathrm{d} x\right)^{r} \\
& \leq \frac{C\left(r, d_{0}, t_{0}, t\right)}{r !}\left(\sqrt{2 t+3-\left(d_{0}+r\right)}+2 / 3\right)^{r} \\
& \leq \frac{C\left(r, d_{0}, t_{0}, t\right)}{r !}(2 t)^{r / 2} e^{-\frac{r}{2}} \frac{d_{0}+r-3}{2 t} e^{\frac{2 r}{3 \sqrt{2 t-\left(d_{0}+r\right)+3}}} \\
& \leq\left(\begin{array}{c}
d_{0}+r-1 \\
d_{0}-1
\end{array}\right)\left(\frac{2 t_{0}+3}{2 t+1}\right)^{d_{0} / 2}\left[\left(1-\frac{d_{0}}{2 t_{0}+3}\right)^{-\left(1-\frac{d_{0}}{2 t_{0}+3}\right)}\right]^{t_{0}+3 / 2} \times \\
& \left(\frac{2 t}{2 t+1}\right)^{r / 2} \exp \left(-\frac{d_{0}}{2}+\frac{\left(d_{0}+r\right)^{2}}{8 t+4}+\frac{\left(d_{0}+r\right)^{3}}{4(2 t+1)^{2}}-\frac{r\left(d_{0}+r-3\right)}{4 t}+\frac{2 r}{3 \sqrt{2 t+3-\left(d_{0}+r\right)}}\right)
\end{aligned}
$$

By removing the $o(1)$ terms in the exponential and using the fact that $x^{-x} \leq e$ we obtain the following bound on the probability $p^{*}$.

$$
p^{*} \leq\left(\begin{array}{c}
d_{0}+r-1 \\
d_{0}-1
\end{array}\right)\left(\frac{2 t_{0}+3}{2 t+1}\right)^{d_{0} / 2} e^{\frac{3}{2}+t_{0}-\frac{d_{0}}{2}+\frac{2 r}{3 \sqrt{t}}} .
$$

\section{Lemma 11}

Proof. Let $\mathcal{A}_{4}$ denote the event that there are two vertices among the first $t_{1}$ with degree $t_{0}^{-1} t^{1 / 2}$ and within $\frac{\sqrt{t}}{f(t)}$ of each other. By the definition of conditional probability and Lemma 8

$$
\operatorname{Pr}\left[\mathcal{A}_{4}\right]=\operatorname{Pr}\left[\mathcal{A}_{4} \mid \overline{\mathcal{A}}_{3}\right] \operatorname{Pr}\left[\overline{\mathcal{A}}_{3}\right]+\operatorname{Pr}\left[\mathcal{A}_{4} \mid \mathcal{A}_{3}\right] \operatorname{Pr}\left[\mathcal{A}_{3}\right] \leq \operatorname{Pr}\left[\mathcal{A}_{4} \mid \overline{\mathcal{A}}_{3}\right]+o(1)
$$

it suffices to show that $\operatorname{Pr}\left[\mathcal{A}_{4} \mid \overline{\mathcal{A}_{3}}\right]=o(1)$. Note that by a simple union bound

$$
\operatorname{Pr}\left[\mathcal{A}_{4}\right] \leq \sum_{1 \leq s_{1}<s_{2} \leq t_{1}} \sum_{l=-\frac{\sqrt{t}}{f(t)}}^{\frac{\sqrt{t}}{f(t)}} p_{l, s_{1}, s_{2}}=O\left(t_{1}^{2} \frac{\sqrt{t}}{f(t)} \max p_{l, s_{1}, s_{2}}\right)
$$

where $p_{l, s_{1}, s_{2}}=\operatorname{Pr}\left[d_{t}\left(s_{1}\right)-d_{t}\left(s_{2}\right)=l \mid \overline{\mathcal{A}}_{3}\right]$.

We consider two cases and we show that in both cases $\max p_{l, s_{1}, s_{2}}=o\left(\frac{f(t)}{t_{1}^{2} \sqrt{t}}\right)$.

- CASE $1\left(s_{1}, s_{2}\right) \notin E\left(G_{t}\right)$ :

Note that at time $t_{1}$ there exist $m_{t_{1}}=3 t_{1}+3<4 t_{1}$ edges in $G_{t_{1}}$. 


$$
\begin{aligned}
p_{l, s_{1}, s_{2}} & \leq \sum_{r=t_{0}^{-1} t^{1 / 2}}^{t_{0}^{1 / 6} t^{1 / 2}} \sum_{d_{1}, d_{2}=3}^{4 t_{1}} \operatorname{Pr}\left[d_{t}\left(s_{1}\right)=r \wedge d_{t}\left(s_{2}\right)=r-l \mid d_{t_{1}}\left(s_{1}\right)=d_{1}, d_{t_{1}}\left(s_{2}\right)=d_{2}\right] \\
& \leq t_{0}^{1 / 6} t^{1 / 2} \sum_{d_{1}, d_{2}=3}^{4 t_{1}}\left(\begin{array}{c}
2 t_{0}^{1 / 6} t^{1 / 2} \\
d_{1}-1
\end{array}\right)\left(\begin{array}{c}
2 t_{0}^{1 / 6} t^{1 / 2} \\
d_{2}-1
\end{array}\right)\left(\frac{2 t_{0}+3}{2 t+1}\right)^{\left(d_{1}+d_{2}\right) / 2} e^{\frac{3}{2}+t_{1}+\frac{2 t_{0}^{1 / 6}}{3}} \\
& \leq t_{0}^{1 / 6} t^{1 / 2} \sum_{d_{1}, d_{2}=3}^{4 t_{1}}\left(2 t_{0}^{1 / 6} t^{1 / 2}\right)^{d_{1}+d_{2}-2}\left(\frac{2 t_{0}+3}{2 t+1}\right)^{\left(d_{1}+d_{2}\right) / 2} e^{2 t_{1}} \\
& \leq t_{0}^{1 / 6} t^{1 / 2} e^{2 t_{1}} t_{1}^{2}\left(2 t_{0}^{1 / 6} t^{1 / 2}\right)^{8 t_{1}-2}\left(\frac{2 t_{0}+3}{2 t+1}\right)^{4 t_{1}} \\
& =t_{0}^{4 t_{1} / 3+1 / 6} t^{-1 / 2} e^{2 t_{1}} t_{1}^{2} 2^{8 t_{1}}\left(2 t_{0}+3\right)^{4 t_{1}}\left(\frac{t}{2 t+1}\right)^{4 t_{1}} \\
& =o\left(\frac{f(t)}{t_{1}^{2} \sqrt{t}}\right)
\end{aligned}
$$

Note that we omitted the tedious calculation justifying the transition from (2) to (3) since calculating the upper bound of the joint probability distribution is very similar to the calculation of Lemma 7

- CASE $2\left(s_{1}, s_{2}\right) \in E\left(G_{t}\right)$ :

Notice that in any case $\left(s_{1}, s_{2}\right)$ share at most two faces (which may change over time). Note that the two connected vertices $s_{1}, s_{2}$ share a common face only if $s_{1}, s_{2} \in\{1,2,3\}$ Consider the following modified process $\mathcal{Y}^{\prime}$ : whenever an incoming vertex "picks" one of the two common faces we don't insert it. We choose two other faces which are not common to $s_{1}, s_{2}$ and add one vertex in each of those. Notice that the number of faces increases by 1 for both $s_{1}, s_{2}$ as in the original process and the difference of the degrees remains the same. An algebraic manipulation similar to Case 1 gives the desired result.

\footnotetext{
${ }^{3}$ We analyze the case where $s_{1}, s_{2} \geq 4$. The other case is treated in the same manner.
} 